\title{
ESPAÑA Y EL NORTE DE ÁFRICA (SIGLOS XV-XVI) EN LA REVUE AFRICAINE: \\ UN REPERTORIO BIBLIOGRÁFICO
}

\author{
Souhila Markria \\ Universidad de Mostaganem
}

\begin{abstract}
Resumen
En este artículo pretendemos reunir la bibliografía específica sobre España y el norte de África aparecida en la publicación histórica Revue Africaine, como material valioso dentro de la producción historiográfica. El conjunto de trabajos permite tener una visión global sobre la ocupación de la franja costera septentrional africana por parte de España y las relaciones entre la Monarquía hispánica y el norte de África durante la Edad moderna.

Palabras clave: Revue Africaine, España, norte de África, repertorio bibliográfico, historiografía.

Abstract

In this article we intend to gather the specific bibliography on Spain and North Africa that appeared in the historical publication Revue Africaine, as valuable material within the historiographic production. The collection of works allows us to have a global vision of the occupation of the northern coastal strip of Africa by Spain and the relations between the Hispanic Monarchy and North Africa during the modern age.
\end{abstract}

Keywords: Revue Africaine, Spain, North Africa, bibliographic repertory, historiography.

\section{INTRODUCCIÓN}

El fin del último baluarte musulmán occidental y la desaparición de Granada como sociedad islámica permitieron a los Reyes Católicos, Fernando e Isabel, establecer una política expansionista fuera de sus confines naturales. La conquista del norte de África fue un operativo inevitable de la Reconquista, y de la política exterior hispana durante el siglo XVI, solamente que el escenario bélico se desplazó hacia tierras africanas. En una línea similar, el Magreb va a ser laboratorio de la política colonial española. La voluntad manifiesta de Isabel la Católica en este espacio geográfico del otro lado del Mediterráneo así lo dictó. Se debía dominar Berbería: 
La Berbería coincide geográficamente con lo que conocemos en la actualidad como el Magreb. [...] Su territorio habitado abarcaría desde Trípoli, en el Mediterráneo Central, hasta Santa Cruz de Cabo Gue (Agadir) y las Canarias, en el Atlántico'.

La rápida política expansiva de los osmanlíes tras la caída de Constantinopla por las tierras del Danubio y la rendición de una gran parte de Asia convierten rápidamente la estrategia política, el equilibrio de poderes y el carácter de las fronteras de los credos religiosos. Por otra parte, durante el siglo XVI, el desarrollo de la actividad corsaria, tanto cristiana como musulmana, tomó la calidad de antagonismo militar y de fructífero negocio.

La conquista del norte de África, la toma del gran puerto de Mazalquivir, los diferentes relatos de los cautivos políticos, las alianzas diplomáticas, la vida propia de los soldados, los avisos que los espías mandan de lo que sucede en la Berbería central, influyeron en la calidad y cantidad de la historiografía europea. El tema de la empresa africana en la Edad moderna no es menor, aunque se ha centrado mucho en los hechos concretos, en especial bélicos y diplomáticos, razones de conquista del otro lado del estrecho de Gibraltar.

En este caso, nuestro objetivo primordial es agrupar los artículos encontrados en la Revue Africaine relacionados con la conquista del litoral africano durante los dos siglos, XV y XVI, con la historia de Argel, con las relaciones entre los dos mundos: el norte de África y la Monarquía hispánica, como repertorio imprescindible para cualquier estudio histórico analítico sobre dicho tema ${ }^{2}$. Revue Africaine fue creada en 1856 y su último número apareció en 1962. Su primer director fue A. Berbrugger y como publicación ejerció durante muchas décadas una influencia decisiva en los estudios sobre la historia y la cultura norteafricanas.

\section{RAZONES DE LA CONQUISTA}

1 Diego Téllez Alarcia, "El papel del Norte de África en la política exterior España (ss. Xv-Xvi)", Revista Espacio, Tiempo y Forma, 2000, vol. 13, pp. 385-420.

2 Véase un repertorio importante y general sobre esta materia en Mercedes García-Arenal, Miguel Ángel de Bunes y Victoria Aguilar, Repertorio bibliográfico de las relaciones entre la península ibérica y el norte de Africa (siglo XV-XVI). Fuentes y bibliografía, Madrid, CSIC, 1989. 
La historiografía africanista se ha interesado de un modo bastante intenso por analizar las razones de la conquista del norte de África, y por extensión los resultados y la evolución de la empresa africana. Como se ha indicado, una parte de la historiografía conviene en que los intereses de la política africana, de modo sintético, fueron económicos: el control de las rutas con el interior, la pesca, las parias, el dominio militar preventivo contra corsarios berberiscos, geoestrategia, ideológicos para mantener el prestigio de la monarquía. Todos estos aspectos desembocaban en el enfrentamiento religioso como aspecto esencial de la conquista territorial ${ }^{3}$.

\section{LA OCUPACIÓN RESTRINGIDA DEL ESPACIO NORTEAFRICANO}

Matizando estas aspiraciones destaca una ocupación restringida, más allá del estrecho de Gibraltar, una ocupación de una franja costera septentrional para establecer: "una marca de vigilancia por medio de ciudades fortificadas al otro lado de una frontera natural, como es el Mediterráneo". Pero en primer lugar, Fernando e Isabel fueron obligados a firmar un tratado con los portugueses después de un largo enfrentamiento: Alcoçovas (I479), en el cual Portugal y España se repartían las zonas de influencia en el Atlántico: el reino de Fez y Guinea para Portugal, el reino de Tremecén para Castilla ${ }^{5}$. Pero las costas del Sahara, entre el cabo Aguer y el de Bojador, frente a las Canarias, dieron lugar a muchos requerimientos: "De Canarias para abaxo contra Guinea"'. Además, el tratado de Tordesillas celebrado entre Castilla y Portugal el 7 de junio de I494 estableció un reparto de tierras y mares, así como zonas de influencia en beneficio de Portugal y Castilla, es decir una expansión castellana de Cádiz a Nápoles:

3 Miguel Ángel de Bunes, La imagen de los musulmanes y del norte de África en la España de los siglos XVI-XVII, Madrid, CSIC, 1989, p. 3.

4 Miguel Ángel de Bunes, “Orán, primera frontera hispano-turca del Mediterráneo”, en Ismet Terki-Hassaine, Emilio Sola Castaño, Alejandro R. Díez Torre y Manuel Casado Arboniés (eds.), Las campanas de Orán, 1509-2009. Estudios en homenaje a Fatima Benhamamouche, Alcalá de Henares, Universidad de Alcalá, 2012, pp. 55-66.

5 Luis de Mármol Carvajal divide el reino de Tremecén en cuatro provincias (Tremecén, Túnez, Argel y Bujía), pero Diego de Suarez Montañéz la divide en: "La misma occidental, la misma de Tremecen de esta celebre ciudad deste nombre, la segunda provincia, siguiendo contra Oriente, es la de Oran, la tercera de Tenes, la cuarta de Argel, la quinta la de Bujia, mas oriental y vecina del rey de Túnez". Citado por Miguel Ángel, de Bunes Ibarra, ob. cit., 1989, p. 22.

6 Juan Eslava Galán, Los Reyes Católicos, Madrid, Booket, 1996, p. 159. 
E1 Tratado de Tordesillas no sólo decide el reparto del territorio africano entre portugueses y castellanos, sino también de otro ámbito más significativo, el océano Atlántico, mediante una línea situada a 370 leguas al oeste de las islas de Cabo Verde. Se reservaban a Castilla y Portugal las islas situadas al oeste y este, respectivamente, de dicha línea ${ }^{7}$.

Así pues, para conseguir algunas bases militares y puntos clave en el litoral mediterráneo africano, resulta interesante crear una infraestructura de contactos y enviar espías con la misión de organizar y hallar las posibilidades de ocupación. El espía escogido para proponer datos y noticias del país africano fue el caballero y regidor de Alcalá don Lorenzo de Padilla, que fue enviado por Alonso de Aguilar al reino de Tremecén. Este último atravesó Orán y anduvo por numerosas localidades del reino de Tremecén en un espacio de un año, espiando y escribiendo sobre las fortificaciones de los pueblos, la disposición de las tierras, sus recursos, el estado de sus castillos y guarniciones y otros que era preciso saber para poder conquistar.

Más tarde en I494, los Reyes Católicos enviaron a otro espía, Hernando de Zafra, para buscar a caudillos norteafricanos que se mostraran con intenciones rebeldes hacia el emir de Tremecén, una escena muy similar a la llevada a cabo contra los granadinos. Este último extiende una hábil red de espionaje, cuyos hilos se prolongan desde Melilla hasta Orán, "Buscan aliados entre los mismos moros de estas ciudades y desarrolla una labor diplomática tan intensa y acertada, que parece mentira que la estemos contemplando a cuatro siglos y medio de distancia".

Por ejemplo en una carta escrita el I4 de enero de I494, Hernando de Zafra afirmaba que muy pocos musulmanes españoles se detenían en el reino de Tremecén, y la mayor parte de ellos buscaban refugio en las grandes ciudades, así: "todo el reino de Tremcén está temblando y con las llaves en la mano" . En otra carta enviada a Isabel, Zafra escribía que ha llegado a hacer una negociación con un moro que prometía la entrega de

7 Eufemio Lorenzo, Francisco Gallego, Gloria Tejedor, El tratado de Tordesillas, Madrid, Anaya, 1995, p. 3.

8 Gregorio Sánchez Doncel, Presencia de España en Orán (1509-1792), Toledo, Estudio Teológico de San Ildefonso, 1991, p. 124.

9 Luis Suarez Fernández, España de los Reyes Católicos, 1474-1516, Madrid, Espasa Calpe, 1992, p. 325. 
Orán en las mismas condiciones de capitulación que las de Granada ${ }^{10}$. Por todo lo comentado, podríamos compartir la opinión de Luis Suarez sobre Hernando de Zafra ${ }^{11}$.

De esta manera, a la luz de los avisos que estaba recogiendo Zafra, las codicias expansionistas españolas en Marruecos no retrocedieron y Melilla (Mililia) fue conquistada por las huestes españolas del jerezano Pedro de Estopiñán, capitán del duque de Medina Sidonia en I497. Melilla, como ya es bien sabido, fue terminal de las caravanas que traían el oro de Sudán a través del Sahara. Su conquista fue también debida a factores estratégicos, por ser un buen lugar costero, fácil de proteger y de suministrar por mar, y para adelantarse hacia el núcleo del sultanato de Fez. Así pues, Melilla reaparecerá como una línea defensiva de avance para proteger la monarquía hispánica de una contraofensiva musulmana.

La voluntad manifiesta de la reina Isabel no se cumplió hasta ahí, sino buscó también otros sitios estratégicos que protegieran las costas españolas de los ataques berberiscos, para que no se produjeran insurrecciones musulmanas en la península Ibérica, zona recién reconquistada. Así, Isabel y Fernando llegaron a ocupar puertos y localidades, y no reinos, o toda una región. De hecho, la presencia española se hallaba restringida a una pequeña parte del litoral mediterráneo africano: "una ocupación restringida del territorio" 12 .

A poco de morir Isabel la Católica, Alcaide de los Donceles preparó una fuerte escuadra reunida en Málagapara la ocupación del amplio puerto de Mazalquivir (Mers el-Kébir) ${ }^{13}$. El I5 de septiembre de 1505, los

10 Gregorio, Sánchez Doncel, ob. cit., p 124.

11 Luis, Suarez, Fernando el Católico, Barcelona, Ariel, 2004, p. 254.

12 Esta término fue empleada por varios historiadores como Fernand Braudel, M. D. de Grammont o Roberd Ricard y últimamente por Beatriz Alonso Acero. Citado por Beatriz Alonso Acero, Orán y Mazalquivir en la política norteafricana de España, 1589-1639, Madrid, UCM, 1997, p. 5. "Portugais, espagnols, Anglais n'ont occupé que des places maritimes, qui demeuraient quelque peu en marge du pays et qui ne pouvaient servir de base à une vraie pénétration", en R. Ricard, "Les établissements européens en Afrique du nord du xv siècle au XvinI siècle et la politique d'occupation restreinte » Revue Africaine, 79, 1938, p. 687.

13 Gregorio Sánchez Doncel, ob.cit.,pp. 125-126. “6 galeras de Aragón y multitud de carabelas andaluzas: 6 fustas, 35 fustas y bergantines, 10 barcos diversos, 6 para carga y descarga, 8 naos, 6 carabelas y 4 tafureas, además de 2" carabelas de Andalucía, 23 de bergantines y 42 navíos menores. las fuerzas navales irían al mando de Don Ramón de Cardona, almirante de Aragón, y las de tierra, unos 7000 hombres, al de don Diego Fernández de Córdoba, asistidos de los esforzados guerreros 
españoles se apoderaron de Mazalquivir y pusieron sus huestes en esta plaza, situada enfrente de Cartagena. Nuevamente, en el mismo año de I505 se tomó la ciudad vecina de Melilla Cazaza conocida antes del siglo Xvi por Kudiat al Bayda. En I505, el duque de Medina Sidonia don Guzmán ordenó las tropas del gobernador de Melilla para sorprender la plaza. España eligió esta zona por su ubicación estratégica y por poseer un gran puerto, como lo menciona bien Luis el Africano: "Cazaza posee un gran puerto donde acostumbraban venir las galeras venecianas que mantenian un importante comercio con las gentes de Fez y proporcionaba buenas ganancias" ${ }^{14}$.

La estrategia hispana de guardar emplazamiento en el litoral africano en 1508 fue continuada con la toma del peñón de Vélez de la Gomera, punto de partida de la ofensiva española sobre los puertos del Magreb. Las reñidas discusiones de Fernando con su yerno portugués terminaron con un acuerdo firmado en el convenio de Sintra, el i8 de septiembre de I509, por el cual se fijaba que toda la costa desde allí a Melilla quedaban en posesión castellana, mientras la costa africana frontal a Canarias, entre los cabos Bojador y Num, con la excepción de Santa Cruz de la Mar Pequeña, quedaba como posesiones portuguesas:

E1 Tratado de Sintra supone nuevamente para España la renuncia dolorosa a futuras expansiones por la zona atlántica africana, que poco a poco y de forma intermitente se había pretendido mantener. Toda África occidental se vendió, exceptuada la Torre de Santa Cruz de Mar Pequeña, a cambio de Peñón de Vélez de la Gomera ${ }^{15}$.

Un año después, el 27 de mayo de I509, Pedro Navarra con la participación personal y material (aliento, propaganda y dinero) del cardenal Cisneros conquistaron un enclave medular en la costa argelina: Orán. Sea como fuere, y como se indicó, la plena gloria de la conquista fue gracias a expertos y personas que tenían experiencia en asuntos de guerra, como Gonzalo de Ayora. Así en un escrito dirigido al rey Fernando, Cisneros explicó cómo llegaron a sorprender Orán gracias al puerto de Mazalquivir: "en fin mataronlos y cativarolons todos, que serán los muertos y

Gonzalo de Ayora y Pedro López de Orozco...”.

14 Enrique Gozalbes. "Notas sobre Cazaza, puerto de Fez y fortaleza española, (1506-1533)", en Al Ándalus-Magreb, 2008, nún. 15, pp. 135-155.

15 Eufemio Lorenzo et al., ob. cit., p. 87. 
cativos más de doce mil moros, ocho los vivos, y más de quatro mill los muertos por las calles y casas" 16 .

Además señaló su miramiento hacia la ciudad: "La cibdad es la más fuerte cosa del mundo, y muy grande, y las más fresca de aguas y huertas y casas que ay en España, y digo a vuestra merced que es más fuerte que Toledo [...] el mas excelente lugar que se vio en el mundo"17.

Más tarde, Pedro Navarra se apoderó de la pequeña isla de Mesranna o el peñón de Argel, como lo llamaron los españoles (I5IO-I529), una fortificación importante para el control del corso berberisco, Bugía y Trípoli. Pero, esta línea de reforzamiento y este rápido avance se detuvo en el desastre de los Gelves, debido a la sequía del verano, la falta de agua y la inexperiencia de don García de Toledo. El poeta Garcilaso de la Vega en unos versos referentes a la jornada dijo:

¡Oh patria lagrimosa, y como vuelves

Los ojos a los Xelves, suspirando! ${ }^{18}$

\section{REPERTORIO BIBLIOGRÁFICO}

1. Berbrugger, A., "Conquête d'Oran », Revue Africaine, I866, ıо, pp. 43-50.

En este estudio el historiador Berbrugger nos hace una sinopsis de una parte de la obra de Diego Suárez Corvin Montañés Historia del último Maestro que fue de Montesa y de su hermano Don Felipe de Borja: la manera como gobernaron las plazas de Orán y Mazalquivir, reinos de Tremecén y Ténez en Africa, siendo alli capitanes generales, uno en pos del otro como aquí se narra. Se trata de la monumental obra de un soldado que vivió en Orán durante casi treinta años (1577-1604). Además. Berbrugger rescata otros documentos sobre la conquista de la ciudad de Orán de I509 y Mer-el Kebir, según Mármol Carvajal (de hecho, el mejor estudio clásico de la historiografía europea sobre el ámbito geográfico del norte de África es su Descripción General de África $\left.{ }^{19}\right)$.

16 AA. VV., Cartas del cardenal don Fray Francisco Jiménez de Cisneros, dirigidas a Don diego López de Ayala, Madrid, Impr. del Colegio de sordomudos y de ciegos, 1867, p. 48.

17 Ibid.

18 Jesús Sánchez Adalid, La sublime Puerta, Barcelona, Zeta, 2005, p. 196.

19 Dentro de la amplia producción histórica sobre este ámbito geográfico se destaca a Mármol 
2. Berbrugger, A, « Etude biographique. Léon L'Africain », Revue Africaine, 1857-1858, 2, pp. 553-364.

El autor nos hace una biografía más o menos fidedigna de Juan León Africano ${ }^{20}$ que ocupa la primera plaza en esta galería africana basándose en su primer editor Giov. Battista Ramusio: "C'est dans cet esprit et avec ces éléments que nous avons écrit la biographie quion va lire."

3. Berbrugger, A, « Negociation entre Hssan Aga et le comte d'Alcaudete, gouverneur d'Oran (154I-I542) », Revue Africaine, 1865, 9, pp. 379-385.

En este texto Berbrugger nos revela tres cartas de la biblioteca de la Secretaria General, en las cuales se describes las negociaciones antes de la expedición argelina de Carlos V de 154r:

Après la lecture des trois lettres que nous venons de donner, il est impossible de contester la réalité des négociations de Hassan Pacha avec les Espagnols dans les années 1540, I54I et I542. Il reste à savoir si elles étaient sérieuses et si le gouverneur d'Alger, à l'exemple de son patron Kheir edDin, le deuxième Barberousse, ne cherchait pas à amuser son ennemi ${ }^{22}$.

4. Berbrugger, A., « Mers-el-Kebir et Oran, de 1509-1608, d'après Diego Suarez Montanés », Revue Africaine, 1865, 9, pp. 251-267, 337- 355 y 4IO- 429; I866, IO, pp. III- I28 y 197-207; II, I867, pp. 72-8I.

En este artículo el autor nos presenta las acciones bélicas de ataque cerca de Mazalquivir, según el propio soldado Diego Suárez Montanés: "Ici, s'interrompt brusquement le chapitre que Suarez a consacré à la guerre d'Oran; par bonheur, la lacune ne peut être ni grande ni importante" 23 .

5. Berbrugger, A, «Oran sous les espagnoles, Expéditions et razzias », Revue Africaine, I869, I3, pp. I00-II5.

El autor nos hace una traducción de dos documentos oficiales contemporáneos a la toma de Mers al Kabir en 1505, recogidos por el general de Sandoval:

como el máximo experto, porque estaba presente en algunos acontecimientos que rememora y llega a conocer bastante bien en el marco geográfico reseñado. Los dos primeros tomos de su libro fueron impresos en Granada en 1573.

20 Llamado antes de su conversión al cristianismo como al-Hasan b. Muhammad al-Wazzan al-Fasi al-Garnati.

21 A. Berbrugger, loc.cit. p. 354.

22 Berbrugger, "Negociation entre Hssan...", art. cit., p. 25.

23 Berbrugger, "Mers -el-Kebir...," art. cit., p. 81. 
Depuis la publication du récit de cette expédition qui livra aux Espagnols un point important du 'littoral algérien, qu'ils conservèrent pendant près de trois siècles, nous avons reçu de M. le Général de Sandoval deux documents officiels contemporains de l'événement, ce qui en double la valeur, ils offriront un précieux moyen de contrôle du récit de Suarèz et de ceux des autres écrivains qui en ont parlée. En voici la traduction $[\ldots]^{24}$.

6. Berbrugger, A, y D. Monnereau « Topographie et Histoire générale d'Alger, par Haedo, traduit de l'espagnol », Revue Africaine, I870, I4, pp. 364-375.

Traducción francesa del cuarto capítulo de la Topografía de Diego Haedo (cuando Argel cayó bajo la dominación de los turcos), según un manuscrito de Argel.

7. Bodin, Marcel, «L'agrément du lecteur/Notice historique sur les Arabes soumis aux Espagnols pendant leur occupation d'Oran, par Si Abd-el-Kader el Mecherfi ", Revue Africaine, 1924, 65, pp, 193-260.

El autor nos hace una traducción del texto de Sidi 'Abdelkader Ben 'Abdallah ben Abī Jalāl al-Mašarfī y Garīsī, obra que nos facilita una lista de las tribus aliadas de España, llamada Babŷat al-nāzir fì ajbār al-dājìīn tahta wilāyat al-isbāniyīn bi-Wabrān mina al-a'rāb kabanì 'āmir:

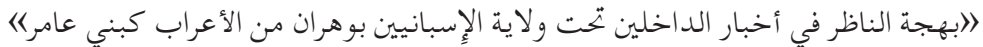

8. Braudel, Fernand, « Les espagnols et l'Afrique du Nord de I492 à I577 ", Revue Africaine, 1929, 69, pp. 184-233; 351-410.

Es un estudio cronológico muy definido para conocer los grandes detalles de la conquista española del norte de África en este período. Es un estudio imprescindible para conocer los principales datos, motivos y efectos de esta estrategia norteafricana siguiendo una periodización histórica referente a la política exterior española: "Ces événements sont d'importance fort inégale et se répartissent sur un demi-siècle à peu près. Là encore, les multiples travaux publiés permettent de dresser facilement un résumé chronologique des principaux événements" 25 .

9. Cazenave, J., «Contribution à l'histoire du vieil d'Oran, Mémoire sur l'état et la valeur des places d'Oran et de Mer-el-Kebir », Revue Africaine, 1925, 66, pp. 323-368.

24 Berbrugger, “Oran sous les espagnoles...”, art. cit., p. 100

25 Braudel, Fernand, “Les espagnols...”, art. cit., p. 351. 
Traducción de un manuscrito inédito de la B.N.P (Biblioteca nacional de París núm. 365, fol. 2II a 232). Escrito por el comandante general ${ }^{26}$ de Orán y Mazalquivir D. José Vallejo en el año I734 después de unas semanas de su actividad militar. El comandante describe las plazas fuertes de la zona de posesión española, los tratos de alianzas, razzia, impuestos, repartición y venta del botín y la estrategia usada en su época con la población local.

10. Cazenave, J., « Les gouverneurs d'Oran pendant l'occupation espagnoles de cette ville (I505-I792), Revue africaine, I930, 71, pp. 257-299.

Monografía que presenta una lista completa de los gobernadores de Orán y Mazalquivir (empezando por el Cardenal Jiménez de Cisneros: el r9 de mayo de 1509 hasta Juan de Courten: el 26 de febrero de i79I), además alude a las gestiones de su administración a lo largo de tres siglos. El historiador usa documentación inédita de Simancas y las bibliotecas nacionales de España y Francia.

11. Devoulx, Alber, "La batterie des Andalous à Algérie ", Revue Africaine, 1872, 16, pp. 340-342.

El autor describe los últimos vestigios de la llamada "batería andalusî" distribuidos en Argel, como recuerdo del Argel otomano:

Le bastion dont je moccupe était placé sur la terre ferme et tout près de la porte de la Marine, laquelle constituait la seule communication entre la ville et le port, formé par des constructions établies sur des îlots et relié à la côte par une jetée d'une longueur d'environ deux cents mètres ${ }^{27}$.

12. Douel, M., «Une information relative à Cervantes, faite a Alger en 1580 ", Revue africaine, I931, 72, pp. 294-318.

Traducción francesa de un texto de Navarrete en su obra Vida de Miguel de Cervantes Saavedra, publicado en i8ı, que está basada en una copia de Simancas relativa a Miguel de Cervantes en 1590. Son ilustraciones y documentos presentados ante el redentor de cautivos Juan Gil en los que Cervantes hace una encuesta con testigos sobre cautividad y su

26 Durante el primer periodo de la ocupación española de Oran, los gobernadores tenían el título de Capitán general del reino de Tremecén y Tenés y gobernador de las plazas de Orán y Mazalquivir. Después de 1732 poseen el grado de de Comandante general. Citado por Cazenave, “Contribution à l'histoire...", art. cit., p. 323.

27 Devoulx, "La batterie des...”, art. cit., p. 340. 
vida en Argel: "il désire et il lui importe de faire une enquête par témoins, tant sur sa captivité, vie et moeurs que sur d'autre choses touchant sa personne"28.

13. Fraude Charles L., "Conquête de Bougie par les Espagnols d'après un manuscrit arabe. (Abū 'Ali Ibrāhīm al-Marīnī. Unwān al-Ajbār) », Revue africaine, 1868, I2, pp. 245-256; 337-349.

El autor traduce este manuscrito árabe conseguido gracias a un personaje de la tribu de Beniyala. Es una descripción de la conquista de Bugía, antes sólo narrada por León Africano y Mármol. Con este nuevo manuscrito, se pueden ver $\mathrm{y}$ distinguir los principales datos de la conquista de Bugía narrados por autores árabes: "Mais ici se présente un fait qui démontre l'utilité de contrôler les documents historiques, en puisant toujours $\grave{a}$ des sources diverses d'informations" ${ }^{29}$.

14. Feraud, Charles L., "Lettres arabes de l'époque de l'occupation espagnole en Algerie », Revue Africaine, I873, 17, pp. 313-321.

Son cuatro cartas árabes de Simancas traducidas en francés, que sirven para profundizar en la historia de la ocupación española de África. La primera carta fue dirigida al Cardenal Jiménez de Cisneros desde Mostaganem alrededor de 1516, la segunda contiene testimonios de fidelidad dirigida al Emperador Carlos V, la tercera dirigida a don Martín de Córdoba en 1535, la cuarta dirigida al conde don Martín por parte de Mohammed Ben Trad y de Ahmed Ben Trad.

15. Feraud, Charles L., " Notes sur Bougie aux diverses époques", Revue Africaine, (1857-58), 2, pp. 458-465 ; 1859, 35, pp. 45-51, 296-308 y 442-468.

El autor nos revela la historia de Bugía desde época romana hasta el siglo xix, y también nos explica la etimología de la palabra "Azuag", derivada de "Azuagues", los primeros habitantes de las montañas de Bugía. "Ce dernier mot signifie Sourd. — Les Iazouguen, dit-on, descendent d'un certain Felen Âzouk- un tel le sourd. - Près de Bougie existe un plateau connu sous le nom de Iril ou Azoug, le plateau du sourd $»^{30}$.

16. Lespes, R., "Oran, ville et port avant l'occupation francaise (I83I)", Revue Africaine, 1934, 75, pp. 277-335.

28 Douel, “Une information...”, art. cit., p. 294.

29 Féraude Charles, “Conquête de Bougie...”, art. cit., p. 246.

30 Feraud, Charles, “Notes sur Bougie...”, art. cit., p. 48. 
Se trata de una historia cronológica de la ciudad de Orán y sus alrededores desde el siglo $\mathrm{x}$ hasta la ocupación francesa aludiendo a su población, su comercio, los viajes: "si nous ne manquons pas de détails sur l'histoire d'Oran du X aux XVI siècle, les renseignements précis sur la ville elle-même sur la population, sur son commerce propre”. Además ofrece unos folios sobre la ocupación española en el siglo XVI.

17. Monchicourd, Charles, «Essai bibliographique sur les plans imprimés de Tripoli, Djerba et Tunis-Goulette au Xvi ${ }^{\mathrm{e}}$ siècle et Note sur un plan d'Alger ", Revue Africaine, I925, 66, pp. 385-418.

Tal y como señala el título, el historiador muestra el interés que había en la época por estas localidades del litoral norteafricano, por las campanas militares y el comercio. Trae un mapa de Trípoli (1567) y otro de Yerba (I560), bien descritos.

18. Monnerreau y E. Watbled, « Négociation entre Charles Quint et Kheir-Eddin (I538-1540) », Revue Africaine, I871, I5, pp. I38-I48.

Se trata de una carta de la Biblioteca del Gobierno General de Argelia enviada a Andrea Doria por el doctor Romero desde Constantinopla para negociar con Jayr al-Dīn: "La pièce la plus curieuse de celle étrange affaire est un rapport détaillé, écrit de Constantinoplé, en 1540, au prince André Doria, par le docteur Romero, qui lui raconte minutieusement ses tribulations politiques avec Barberousse" ${ }^{1}$.

19. Monnerreau y Berbrugger, "Topographie et histoire générale d'Alger, par Haedo, traduit de l'espagnol », Revue Africaine, I870, I4, pp. 364-365, 4I4-433 y 490-519, núm. 66.

Como hemos mencionado anteriormente, es la continuación de una traducción francesa del cuarto capítulo de la Topografía de Haedo (cuando argel cayó bajo la dominación de los turcos), según un manuscrito de Argel.

20. P. P. « L'expédition espagnole de I54I contre Alger », Revue Africaine, I89I, 35, pp. I77-206.

El objetivo de este estudio es hacer una nueva traducción de los escritos relativos a la expedición de Carlos V contra Argel junto con la versión árabe de las Gazawāt, en el que se haya comprendido el relato de la expedición de Argel:

31 Monnerreau y E. Watbled, “Négociation...”, art. cit., p. 140. 
Une autre relation, quon a dénommée manuscrit du Mehkémé, est ajoutée en appendice dans les divers exemplaires arabes des Ghazaouât dont nous avons pu, directement ou indirectement, avoir connaissance (deux manuscrits de la Bibliothèque d'Alger, deux appartenant à des particuliers, un à Paris $)^{32}$.

21. Pavy, Mgr., « La piraterie barbaresque », Revue africaine, (I857I858), 2, pp. 337-352.

El historiador alude a la piratería, cruzadas y postura de la iglesia en los siglos XII-XIX : "Ce fut, depuis l'occupation de l'Algérie par les Turcs, au commencement du XVIe siècle, la métropole des forbans et des martyrs. Que faisait donc l'Eglise? Que faisaient les peuples et leurs gouvernements?"33.

22. Pastemal-Djoglou, A, « Ce qui subsiste de l'Oran espagnol », Revue Africaine, 1936, 79, pp. 665-686.

El historiador A. Pastemal alude a los restos arquitectónicos de la ciudad de Orán española del siglo xviıI y otros algunos del s. Xvi (las fortificaciones, los castillos, murallas, puertas, plazas).

23. Pastemal-Djoglou, A, « Mers-el-Kebir. Historique et description de la forteresse ", Revue Africaine, I940, 84, pp. 154-I85.

Es una historia y descripción de la fortaleza antes de 1563 con figuras y planos, y la del siglo xviII hasta su estado actual.

24. Primaudaie, Élie de la, “ Document inédits sur l'histoire de loccupation espagnole en Afrique (1506-I574) », Revue Africaine, 1877, 21, pp. I7-32, 8I-96, I98-229, 265-298, 36I-379 у 46I-472.

Traducciones de documentos inéditos sobre la ocupación española en dicho período conservados en Simancas acompañados con un análisis. El primer documento es un informe del consejo de su Majestad sobre los asuntos del reino de Tremecén en diciembre de 1535 (AGS: Estado, Legajo 463), el segundo es la situación de artillería, alcazaba vieja (AGS: Estado, Legajo 462), el tercero es una carta de don Bernardino de Mendoza a su Majestad escrita en Goleta-Túnez el 20 de diciembre de 1535 (AGS: Estado, Legajo 463), el cuarto son instrucciones del Conde de Alcaudete a García de Navarrete, comandante de Mazalquivir en 1535 (AGS: Estado, Legajo 463), el quinto es una carta del Conde de Alcaudete a Ben Reḍwān

32 P.P, “L'expédition espagnole...”, art. cit., p. 177-178.

33 Pavy, Mgr., "La piraterie...”, art. cit., p. 342. 
en enero de 1536 (AGS: Estado, Legajo 463), y el último documento es una carta del Conde de Alcaudete a šayj Būziān Sāhị en enero de 1536 (AGS: Estado, Legajo 463).

25. Primaudaie, Elie de la, "Les villes maritimes du Maroc. Commerce, navigation, géographie comparée ", Revue Africaine, I872, I6, pp. 105-135, 2OI-2I5, 304-3I8, 388-400 y 456-470 ; I973,I7, pp. 66-75,I2I-I3I y 275-286.

Descripción y análisis histórico de las ciudades marítimas de $\mathrm{Ma}$ rruecos. Es una evaluación general en la que están incluidos los tiempos de ocupación española y portuguesa y una breve síntesis de los mismos.

26. Ravenent, E, «Un épisode de l'expédition de I54I contre Alger. Léchauffourée de la porte d'Azzoun ", Revue Africaine, I939, 83, pp.303322.

Tal y como señala el título, un episodio de los hechos, entre los cuales está el episodio de la puerta de Azzoun "Bab Azzūn”, el autor alude a la expedición de Argel de r54I:

En face de square Bresson, le second pilier de l'immeuble qui fait l'angle des rues bab Azzoun est Littré porte une plaque de marbre sur laquelle on lit l'inscription suivante: à quelques pas d'ici le 25 octobre I54I, le français Pons de Balaguer dit savignac porte étendard des chevaliers de Malte qui firent partie de l'expédition dirigée par Charles Quint contre Alger ${ }^{34}$.

27. Ricard, Robert, «L'Espagne et la fabrication des 'bonnets tunisiens'. A propos d'un texte du XVIII siècle ", Revue Africaine, I956, Ioo, pp. $423-432$.

En líneas generales, señala la producción del bonete tunecino en $\mathrm{Ma}$ drid el ir de junio de 1787 y su difusión a lo largo del siglo Xvir basándose en un informe encontrado en la obra de Gaspar Melchor de Jovellanos de la Biblioteca de Autores Españoles (BAE).

28. Ricard, Robert, «Les établissements européens en Afrique du Nord du $\mathrm{XV}^{\mathrm{e}}$ au XVIII ${ }^{\mathrm{e}}$ siècle et la politique d'occupation restreinte ", Revue Africaine, 1936, 79, pp. 687-688.

En general, manifiesta la forma de ocupación restringida en el Norte de África (plazas marítimas) por los portugueses y españoles; unos por la costa atlántica y otros por la costa mediterránea. Presenta esta ocupación

34 Ravenent, “Un épisode de l'expédition...”, art. cit., p. 303. 
restringida como una necesidad más que como un sistema político. Además, el historiador alude y compara la ocupación restringida del Magreb por los españoles con la historia de los ingleses en Tánger.

29. Ricard, Robert, « Le Père Jérome Gratien de la Mère de Dieu et sa captivité à Tunisie (1593-I595) ", Revue Africaine, I945, 89, pp. I90-200.

Continuación del anterior artículo sobre los tratos españoles de Berbería en el que ya menciona al padre Jerónimo Gracián de la Madre de Dios que se considera como un testimonio de la vida de los cautivos cristianos a finales del xvi. Era hijo de Diego Gracián de Alderete, secretario de Carlos V y Felipe II.

30. Ricard, Robert, « Textes espagnols sur Berbérie $\left(\mathrm{XV}^{\mathrm{e}}, \mathrm{XVI}^{\mathrm{e}}\right.$ et $\mathrm{XVII}^{\mathrm{e}}$ siècles) ", Revue Africaine, I945, 89, pp. 26-40.

El historiador agrupa algunos textos inéditos sobre Berbería o crónicas generales que contienen capítulos y páginas sobre las relaciones de África y la península Ibérica, analiza cinco de ellos: El victorial de don Pero Niño (1448), el del Jerónimo Gracián ( 1593-I595), el del P. José de Sigüenza ( I60o -I605), el del clérigo P. Pedro Ordóñez de Ceballos (I6I6) en Historia y viaje del mundo del clérigo acrecido D Pedro Ordóñez de Ceballos, natural de la insigne ciudad de Jaén a la cinco partes de Europa África, Asia y América Magallánica, y el del P. José Tamayo (I64I-I643).

31. Veronne, Chantal de la, «Deux lettres inédites d'un roi de Tlemcen (153I-I532) ", Revue Africaine, I955, 99, pp. I74-I80.

Describe dos cartas encontradas en 1954 en una librería parisina. Son cartas respectivamente escritas el 8 de septiembre de I53I y 3 de Julio de I532 por el soberano zianí de Tremecén Muley Abd Allah, mandadas a la emperatriz Isabel de Portugal, que prueban las tentativas del rey de Tremecén por recobrar la alianza española.

32. Watbled, E., « Document inédits sur l'assassinat du pacha Tekelerli (1556-1557) ", Revue Africaine, I871, I5, pp. 335-340.

Entre los numerosos documentos encontrados en la Biblioteca de la Secretaria General del Gobierno sobre la dominación española de África figuran las dos cartas manuscritas que aluden a la muerte del Pacha Mohammed Tekelerli (Tekerli Tekali, Takarouaourli, Takouli), las cuales difieren de las noticias contadas por el historiador Diego Haedo. 


\section{Conclusiones}

Este repertorio bibliográfico ofrece textos históricos, aún hoy valiosos, tanto de fuentes primarias como de interpretaciones históricas, sobre la presencia española en el norte de África, aparecidos en una de las publicaciones más emblemáticas, la Revue Africaine. Gracias a tales trabajos, y las facilidades de acceso que ofrece la actual digitalización del patrimonio bibliográfico, contamos con datos de alto valor académico para analizar uno de los fenómenos más determinantes de la historia norteafricana y sus transformaciones en la Edad moderna. 